\title{
Optically active chiral Ag nanowires
}

\author{
Liguo Ma, Zhehao Huang*, Yingying Duan, Xuefeng Shen and Shunai Che*
}

\begin{abstract}
Chiral Ag nanowires (CAgNWs), fabricated inside chiral carbon nanotubes (CCNTs), exhibit strong circular dichroism (CD) signals in the visible and near-IR regions. Enantiopure CCNTs were prepared by carbonization of the self-assembled chiral polypyrrole nanotubes according to our previous report. Ag ions could be easily introduced into the chiral pores of CCNTs due to the capillary phenomenon. After hydrogen reduction, the optically active CAgNWs formed inside the channels of the CCNTs. The helical channels in the CCNTs played a predominant effect on the chiral formation of the CAgNWs. This system provides new insight into the fabrication as well as the study of optical activity (OA) of chiral inorganic nanomaterials. Such novel chiral inorganic material will bring new opportunities in non-linear optics, biosensors and chiral recognition.
\end{abstract}

\section{INTRODUCTION}

Metallic nanomaterials (nanowires and nanoparticles) are widely considered as potential structural and functional building-blocks for materials in catalysis, separation, optics, and nanoelectronics [1-4]. Many properties of these materials are predicted to be dependent on their size, shape, and spatial arrangement $[5,6]$. Among them, the chirality of metallic nanomaterials receives arising attention because it offers the ability to achieve strong optical activity (OA) over a broad spectral range [7-9]. This is the effect of the difference in speed and absorption of the leftand right-handed circularly polarized light when passing through the chiral materials. Such chiral plasmonic nanostructures provide potential applications in asymmetric catalysts [10,11], optical devices [12] and biological sensors [13-16].

Since Schaaff and Whetten [17] firstly reported the chiral glutathione-protected Au nanoparticles (NPs) exhibiting particular OA in surface plasmon resonance, diverse types of chirality in metal NPs have been found to create plasmonic circular dichroism (CD) [18]. These systems include metal NPs capped with various small chiral molecules, or biological macromolecules such as DNA [19-22], amino acids [23-28], peptides [29-31], and pro- teins [32,33]. Moreover, MacLachlan's group used chiral nanocrystalline cellulose as hard template to prepare chiral nematic cellulose-silver [34] and -gold [35] NP composites. However, these types of chirality in metal NPs rely on the capped chiral molecules, which are unstable under severe conditions, such as high temperature.

Recently, we have obtained chiral arrangement of Ag NPs and nanowire arrays in chiral mesoporous silica by hard template method [36,37], which resulted in the OA in surface plasmon resonance based on chiral superstructure of assembled NPs. However, the origin of the chiral OA by hard template method is so complicated that more efforts are needed to reveal the mechanism.

Herein, we present the synthesis of chiral Ag nanowires (CAgNWs) which features collective intrinsic property of OA by a simple hard template method. Notably, the Ag nanowires are chiral, which are extremely rare [38-41]. For the preparation of ordered nanoarchitecture, hard template method has many advantages, especially in its stability, predictability, controllability and versatility. The synthesis method involves directly casting of Ag ions in chiral carbon nanotubes (CCNTs), subsequent reduction of the metal precursors into CAgNWs in $\mathrm{H}_{2}$. The resulted materials with CAgNWs embedded, which endows them distinct OA, responding to circularly polarized light. To the best of our knowledge, this is the first example of organizing metal into CCNTs with OA, and such method can be extended for preparing other functional metal composites.

As shown in Scheme 1a, the CCNTs were synthesized by the method described in our previous report [42]. Fig. 1a shows the chiral double helix carbon nanotubes. The white colour region in the middle of chiral carbon nanotube stands for helical pores after carbonization in Ar. Then, CCNTs were employed as hard templates to fabricate CAgNWs. Silver ions could be easily introduced into the chiral pores of CCNTs due to the capillary phenomenon. After hydrogen reduction, the CAgNWs were formed inside the channels of the CCNTs by the impregnation of $\mathrm{AgNO}_{3}$ (Scheme 1b). The gray colour region in the middle of chiral

School of Chemistry and Chemical Engineering, State Key Laboratory of Metal Matrix Composites, Shanghai Jiao Tong University, Shanghai 200240, China

*Corresponding authors (emails: chesa@sjtu.edu.cn (Che S); apollo207@sjtu.edu.cn (Huang Z)) 
a

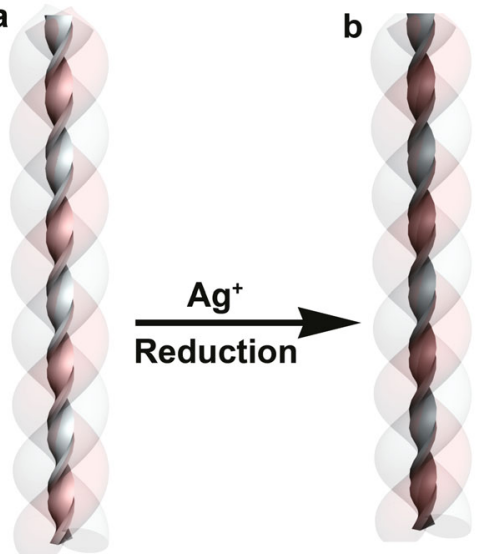

Scheme 1 Schematic illustration of the synthesis of CAgNWs with OA. (a) CCNTs prepared by carbonization of CPPy synthesized by self-assembling of $N$-stearyl-D-glumatic acid $\left(\mathrm{C}_{18}-\mathrm{D}-\mathrm{Glu}\right)$ and pyrrole. (b) CAgNWs prepared from CCNTs.

carbon nanotube in Fig. 1b represents the formation of chiral Ag nanowire. This procedure granted the samples with left- and right-handed helical orientations, which were denoted as L- and R-C-Ag, respectively.

\section{EXPERIMENTAL DETAILS}

\section{Preparation of chiral hard templates of CCNTs}

Firstly, the CCNTs derived from chiral polypyrrole (CPPy) nanotubes by carbonization in an inert gas were synthesized by a modified method according to our previous report [42]. Typically, the molar composition of lipid amphiphilic molecule:ammonium persulfate (APS):pyrrole: methanol:water was 1:40:40:5351:57081. In a typical synthesis, $0.06 \mathrm{mmol}$ of $\mathrm{C}_{18}$-D-Glu was dissolved in $10.2 \mathrm{~g}$ methanol at room temperature, and then $2.4 \mathrm{mmol}$ pyrrole and $60 \mathrm{~mL}$ deionized water were added. The solution was stirred for $10 \mathrm{~min}$, and then a precooled aqueous solution of APS (2.4 mmol in $1.2 \mathrm{~mL}$ deionized water) were added to the mixture and stirred for a further $30 \mathrm{~min}$. A brown solid was obtained after filtrating and thrice-washing with water and ethanol, followed by drying for $12 \mathrm{~h}$ at $40^{\circ} \mathrm{C}$ in vacuum. For the carbonization of the CPPy nanotubes, the synthesized products were heated at a rate of $1.5^{\circ} \mathrm{C} \mathrm{min}^{-1}$ to $550^{\circ} \mathrm{C}$ under Ar flow, for $6 \mathrm{~h}$. After slowly cooling the sample to room temperature, CCNTs were obtained.

\section{Synthesis of CAgNWs@CCNTs}

In a typical synthesis, CCNTs powder $(20 \mathrm{mg})$ was dispersed in an aqueous solution of $\operatorname{AgNO}_{3}\left(2 \mathrm{~mL}, 10 \mathrm{mg} \mathrm{mL}^{-1}\right)$, and then stirred for $30 \mathrm{~min}$ at room temperature. The mixture was reacted statically for $3 \mathrm{~h}$. The water was evaporated slowly in a rotary evaporator and then washed with acetone to remove the Ag ions bounded to the outer surface of the CCNTs. Subsequently, the obtained solids were dried in a vacuum oven at room temperature. The collected samples were placed in a tube furnace, and the metal precursors were reduced under a constant $\mathrm{H}_{2}$ flow at $120^{\circ} \mathrm{C}$ for $6 \mathrm{~h}$. The temperature was increased from room temperature to

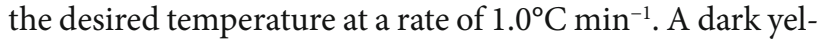
low powder was collected after the tube furnace was cooled to room temperature.

\section{Characterization}

The microscopic characteristics of the samples were observed with JEOL JSM-7401F scanning electron microscope (SEM). Transmission electron microscopy (TEM) observation was performed with a JEOL JEM-2100 microscope operating at $200 \mathrm{kV}$. The X-ray diffraction (XRD) patterns were recorded on a Rigaku D/Max 2000 powder diffractometer equipped with $\mathrm{Cu} \mathrm{K} \alpha$ radiation. The solid-state diffuse-reflectance circular dichroism (DRCD) and diffuse reflectance UV/vis (DRUV/vis) spectra of the powder samples were obtained on a JASCO J-815 spectropolarimeter fitted with a DRCD apparatus.

\section{RESULTS AND DISCUSSION}

Figs $1 a_{1}$ and $b_{1}$ show the SEM images of the CCNTs. These samples are composed of exclusively left- or right-handed double helical fibers with uniform morphology. The outer
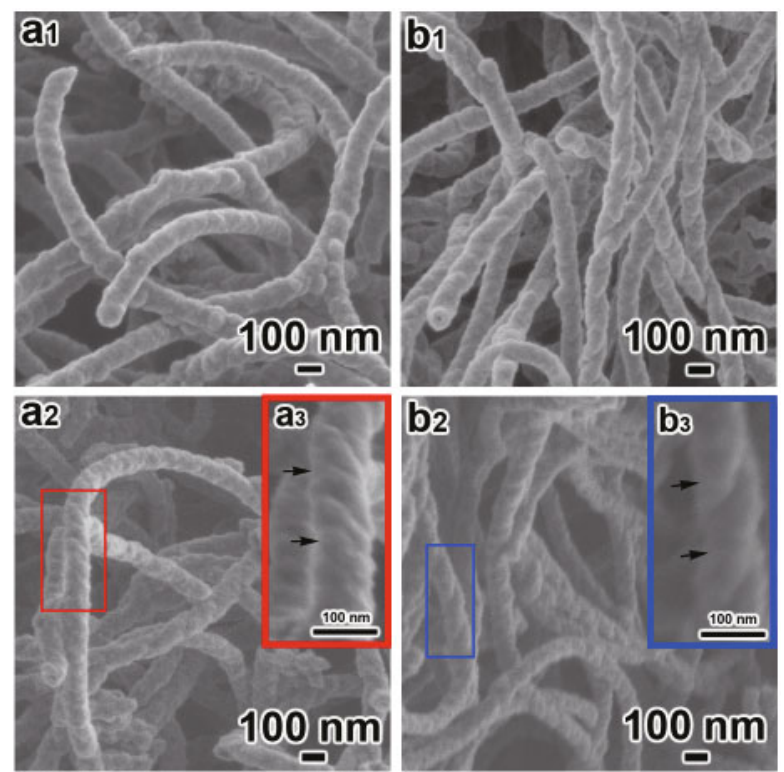

Figure 1 SEM images of the antipodal CCNTs $\left(a_{1}\right.$ and $\left.b_{1}\right)$ and the CAgNWs obtained by reduction in $\mathrm{H}_{2}\left(\mathrm{a}_{2}-\mathrm{a}_{3}\right.$ and $\left.\mathrm{b}_{2}-\mathrm{b}_{3}\right)$. (a, left-hand; $b$, right-hand). 
diameters of the CCNTs are $\sim 100 \mathrm{~nm}$ and the pitch length is estimated to be $\sim 200 \mathrm{~nm}$ (the length between the arrows indicated in Figs $1 a_{3}$ and $b_{3}$ is the pitch length). After the uniform filling of Ag NWs into the CCNTs by hydrogen reduction, the outer diameters slightly decrease due to the shrinkage by hydrogen reduction at high temperature for a long time. However, the double helical morphology is well-maintained even after long-time reduction in $\mathrm{H}_{2}$, which shows the high memory capability of morphology (Figs $1 a_{2}$ and $b_{2}$ ). Figs $1 a_{3}$ and $b_{3}$ are high resolution SEM images of L-C-Ag and R-C-Ag, respectively. Obviously, there is no Ag NPs on the surface of the CCNTs.

Figs $2 \mathrm{a}$ and $\mathrm{b}$ are the TEM images of the Ag NWs within the L-C. Dark, wirelike objects with a uniform diameter of about $10 \mathrm{~nm}$ are observed within the CCNTs, which is smaller than the diameter of the channel ( 20 nm) [42]. When the helical radius is larger than the diameter of single chiral fibers which form double helix, the helical pores will form in the middle of the double helical carbon nanotubes. Along with the shrinkage of the outer diameter, the channels also shrink by hydrogen reduction at high temperature for a long time. Although the single stranded helical morphology of Ag NW inside the CCNT can be clearly observed from the TEM images (Fig. 2b), it is hard to clearly see the pitch length of single stranded helical Ag NW due to the high pitch length/diameter ratio. It can be observed that the Ag NWs are not continuous in some areas because the CCNTs are so long that the Ag ions are unable to fill all
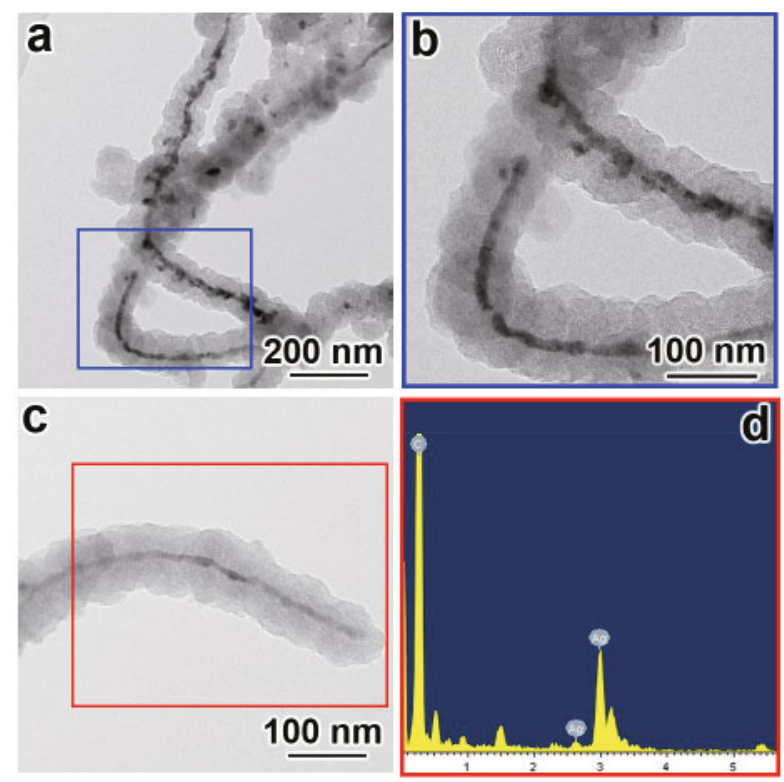

Figure 2 TEM images (a and b) of the L-C-Ag. The L-C-Ag (c) and its corresponding EDX result (d). of the pores. Fig. $2 \mathrm{c}$ shows the single L-C-Ag. As shown in Fig. 2d, the energy dispersive X-ray detector (EDX) analysis of the L-C-Ag indicates that Ag element is in each carbon nanotube. The results suggest that these Ag NWs are oriented in the helical pores.

The XRD pattern (Fig. 3) of the L-C shows a broad diffraction peak at $2 \theta \approx 25^{\circ}$ because the CCNTs are amorphous. The wide-angle XRD patterns of the L-C-Ag reveals four diffraction peaks at $2 \theta=38.1^{\circ}, 44.3^{\circ}, 64.5^{\circ}$, and $77.58^{\circ}$ (Fig. 3), which are assigned to the (111), (200), (220), and (311) reflections of a face-centered cubic silver lattice, respectively.

The CD can be used to measure the differential absorption of left- and right-handed circularly polarized light, and the CD activity indicates a chiral structure, which can be detected by DRCD. Fig. 4 shows the DRCD spectra of two pairs of the antipodal L/R-C and L/R-C-Ag samples. The

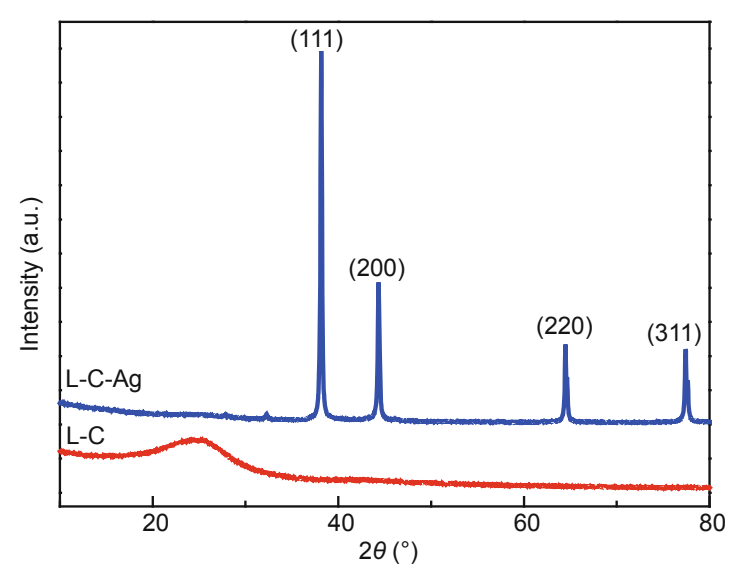

Figure 3 XRD patterns of L-C and L-C-Ag.

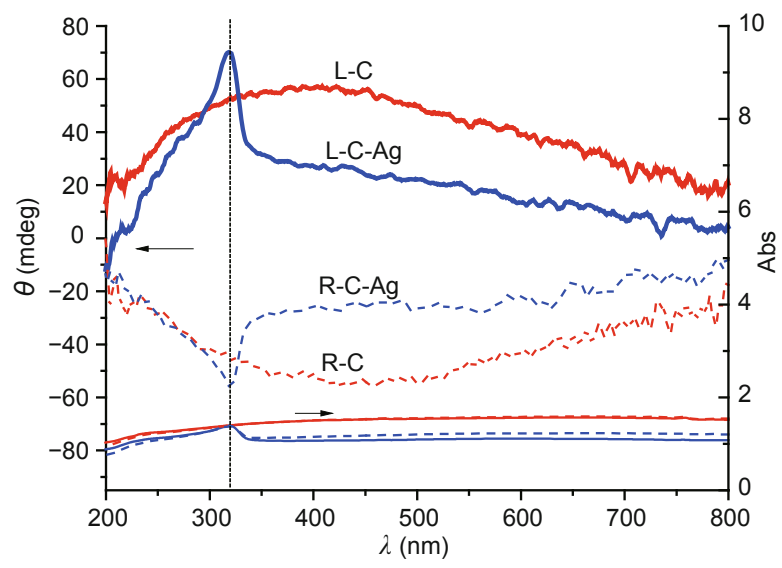

Figure 4 DRUV-vis and DRCD spectra of antipodal CCNTs and CAgNWs. 
antipodal CCNTs show obvious mirror-image CD signals with a peak at $\sim 460 \mathrm{~nm}$, which indicates that the optically active CCNTs can selectively reflect left- or right-handed circularly polarised light in the UV absorption region through a vicinal effect of the helical stacking of carbon nanostructure. A broad absorption band in the range of $350-800 \mathrm{~nm}$ is attributed to the CCNTs, resulting from the combination of nano effect, polarization, and $\pi$-conjugated structure.

For the L/R-C-Ag materials, the DRCD spectra show the overlapped signals attributed to the CAgNWs and CCNTs. L/R-C-Ags show symmetric sharp peaks at the wavelength of $\sim 320 \mathrm{~nm}$ and broad peaks in the range of 350-800 $\mathrm{nm}$. The characteristic band at $320 \mathrm{~nm}$ of L-C-Ag is a result of the absorption of inhomogeneous Ag NWs near the region of the inter-band transitions due to surface plasmon resonance optical signatures of silver nanowires [43-45]. The decreased intensity of broad peak in rang of 350-800 nm also may be due to negative cotton effect on CD signals of $\mathrm{L}-\mathrm{C}-\mathrm{Ag}$ at $350-800 \mathrm{~nm}$ based on the coupled dipole and exciton coupling theory. Moreover, the CD intensity of L-C after reduction in $\mathrm{H}_{2}$ decreases due to partial deformation at high temperature for a long time (as shown in Fig. 5), which leads to the decreased CD intensity in the range of 350-800 nm for L-C-Ag (as shown in Fig. 4).

\section{CONCLUSIONS}

In conclusion, we have successfully prepared chiral Ag NWs with a distinct helix by using CCNTs as hard templates. These novel chiral Ag NWs supported by CCNTs exhibit distinctive plasmon $\mathrm{CD}$ signals, which originates from the collective plasmon coupling of the CAgNWs. It is possible to produce other metallic chiral NWs by using

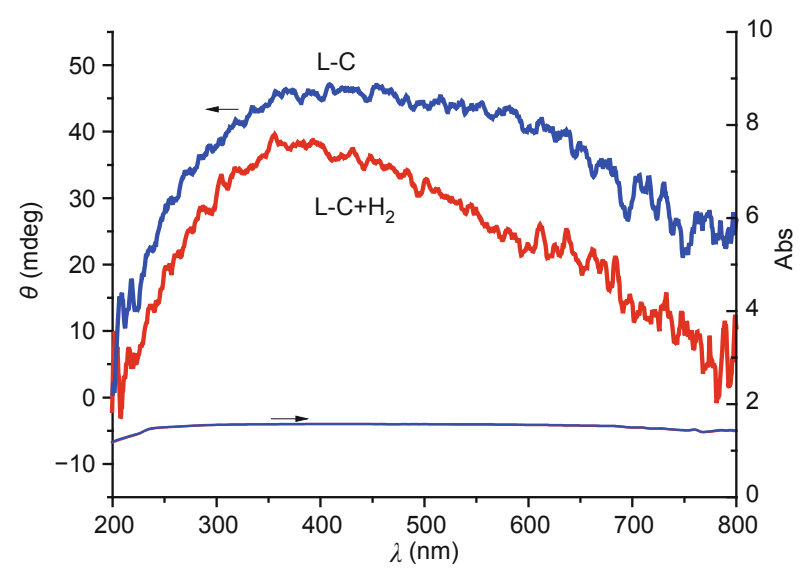

Figure 5 DRUV-vis and DRCD spectra of CCNTs and CCNTs after reduction in $\mathrm{H}_{2}$. metals such as Pt and Au with a similar synthetic procedure. This approach provides the possibility of exploiting the optical and electronic properties of intrinsically chiral metallic compounds for electronics, sensing, and electrochemical applications.

Received 22 May 2015; accepted 8 June 2015;

published online 15 June 2015

1 Hutchings GJ, Haruta M. A golden age of catalysis: a perspective. Appl Catal A-Gen, 2005, 291: 2-5

$2 \mathrm{Hu}$ X, Dong S. Metal nanomaterials and carbon nanotubes-synthesis, functionalization and potential applications towards electrochemistry. J Mater Chem, 2008, 18: 1279-1295

3 Murray RW. Nanoelectrochemistry: metal nanoparticles, nanoelectrodes, and nanopores. Chem Rev, 2008, 108: 2688-2720

4 Schierhorn M, Lee SJ, Boettcher SW, Stucky GD, Moskovits M. Metal-silica hybrid nanostructures for surface-enhanced Raman spectroscopy. Adv Mater, 2006, 18: 2829-2832

5 Leutwyler WK, Bürgi SL, Burgl H. Semiconductor clusters, nanocrystals, and quantum dots. Science, 1996, 271: 933-937

6 Tang Z, Kotov NA. One-dimensional assemblies of nanoparticles: preparation, properties, and promise. Adv Mater, 2005, 17: 951-962

7 Guerrero-Martínez A, Alonso-Gómez JL, Auguié B, Cid MM, Liz-Marzán LM. From individual to collective chirality in metal nanoparticles. Nano Today, 2011, 6: 381-400

8 Valev VK, Baumberg JJ, Sibilia C, Verbiest T. Chirality and chiroptical effects in plasmonic nanostructures: fundamentals, recent progress, and outlook. Adv Mater, 2013, 25: 2517-2534

9 Zhan C, Wang J, Yuan J, et al. Synthesis of right- and left-handed silver nanohelices with a racemic gelator. Langmuir, 2003, 19: 9440-9445

10 Tang Y, Cohen AE. Enhanced enantioselectivity in excitation of chiral molecules by superchiral light. Science, 2011, 332: 333-336

11 Tamura M, Fujihara H. Chiral bisphosphine BINAP-stabilized gold and palladium nanoparticles with small size and their palladium nanoparticle-catalyzed asymmetric reaction. J Am Chem Soc, 2003, 125: $15742-15743$

12 Verbiest T, Rodriguez V. Focus issue introduction: chiral optical materials. Opt Mater Express, 2011, 1: 3-4

13 Hendry E, Carpy T, Johnson J, et al. Ultrasensitive detection and characterization of biomolecules using superchiral fields. Nat Nanotechnol, 2010, 5: 783-787

14 Rosi NL, Mirkin CA. Nanostructures in biodiagnostics. Chem Rev, 2005, 105: 1547-1562

$15 \mathrm{Wu}$ X, Xu L, Liu L, et al. Unexpected chirality of nanoparticle dimers and ultrasensitive chiroplasmonic bioanalysis. J Am Chem Soc, 2013, 135: 18629-18636

16 Liu W, Zhu Z, Deng K, et al. Gold nanorod@chiral mesoporous silica core-shell nanoparticles with unique optical properties. J Am Chem Soc, 2013, 135: 9659-9664

17 Schaaff TG, Whetten RL. Giant gold-glutathione cluster compounds: intense optical activity in metal-based transitions. J Phys Chem B, 2000, 104: 2630-2641

18 Gautier C, Bürgi T. Chiral inversion of gold nanoparticles. J Am Chem Soc, 2008, 130: 7077-7084

19 Shemer G, Krichevski O, Markovich G, et al. Chirality of silver nanoparticles synthesized on DNA. J Am Chem Soc, 2006, 128: 11006-11007

20 Li Z, Zhu Z, Liu W, et al. Reversible plasmonic circular dichroism of Au nanorod and DNA assemblies. J Am Chem Soc, 2012, 134: 3322-3325 
21 Numata M, Sugiyasu K, Hasegawa T, Shinkai S. Sol-gel reaction using DNA as a template: an attempt toward transcription of DNA into inorganic materials. Angew Chem Int Ed, 2004, 116: 3341-3345

22 Kuzyk A, Schreiber R, Fan Z, et al. DNA-based self-assembly of chiral plasmonic nanostructures with tailored optical response. $\mathrm{Na}$ ture, 2012, 483: 311-314

23 Li C, Deng K, Tang Z, Jiang L. Twisted metal-amino acid nanobelts: chirality transcription from molecules to frameworks. J Am Chem Soc, 2010, 132: 8202-8209

24 Sánchez-Castillo A, Noguez C, Garzón IL. On the origin of the optical activity displayed by chiral-ligand-protected metallic nanoclusters. J Am Chem Soc, 2010, 132: 1504-1505

25 Zhu Z, Liu W, Li Z, et al. Manipulation of collective optical activity in one-dimensional plasmonic assembly. ACS nano, 2012, 6: 2326-2332

26 Li Y, Liu M. Fabrication of chiral silver nanoparticles and chiral nanoparticulate film via organogel. Chem Commun, 2008, 43: 5571-5573

27 Han B, Zhu Z, Li Z, Zhang W, Tang Z. Conformation modulated optical activity enhancement in chiral cysteine and au nanorod assemblies. J Am Chem Soc, 2014, 136: 16104-16107

28 Wang X, Duan P, Liu M. Universal chiral twist via metal ion induction in the organogel of terephthalic acid substituted amphiphilic L-glutamide. Chem Commun, 2012, 48: 7501-7503

29 Nishida N, Yao H, Ueda T, Sasaki A, Kimura K. Synthesis and chiroptical study of d/l-penicillamine-capped silver nanoclusters. Chem Mater, 2007, 19: 2831-2841

30 Behar-Levy H, Neumann O, Naaman R, Avnir D. Chirality induction in bulk gold and silver. Adv Mater, 2007, 19: 1207-1211

31 Zhou Y, Zhu Z, Huang W, et al. Optical coupling between chiral biomolecules and semiconductor nanoparticles: size-dependent circular dichroism absorption. Angew Chem Int Ed, 2011, 50: 11456-11459

32 Carmeli I, Lieberman I, Kraversky L, et al. Broad band enhancement of light absorption in photosystem I by metal nanoparticle antennas. Nano Lett, 2010, 10: 2069-2074

33 Slocik JM, Govorov AO, Naik RR. Plasmonic circular dichroism of peptide-functionalized gold nanoparticles. Nano Lett, 2011, 11: 701-705

34 Qi H, Shopsowitz KE, Hamad WY, MacLachlan MJ. Chiral nematic assemblies of silver nanoparticles in mesoporous silica thin films. J
Am Chem Soc, 2011, 133: 3728-3731

35 Schlesinger M, Giese M, Blusch LK, Hamad WY, MacLachlan MJ. Chiral nematic cellulose-gold nanoparticle composites from mesoporous photonic cellulose. Chem Commun, 2015, 51: 530-533

36 Xie J, Che S. Chirality of anisotropic metal nanowires with a distinct multihelix. Chem-Eur J, 2012, 18: 15954-15959

37 Xie J, Duan Y, Che S. Chirality of metal nanoparticles in chiral mesoporous silica. Adv Funct Mater, 2012, 22: 3784-3792

38 Zhu Y, He J, Shang C, et al. Chiral gold nanowires with BboerdijkCoxeter-Bernal structure. J Am Chem Soc, 2014, 136: 12746-12752

39 Kondo Y, Takayanagi K. Synthesis and characterization of helical multi-shell gold nanowires. Science, 2000, 289: 606-608

40 Velázquez-Salazar JJ, Esparza R, Mejia-Rosales SJ, et al. Experimental evidence of icosahedral and decahedral packing in one-dimensional nanostructures. ACS nano, 2011, 5: 6272-6278

41 Wang Y, Wang Q, Sun H, et al. Chiral transformation: from single nanowire to double helix. J Am Chem Soc, 2011, 133: 20060-20063

42 Liu S, Duan Y, Feng X, Yang J, Che S. Synthesis of enantiopure carbonaceous nanotubes with optical activity. Angew Chem Int Ed, 2013, 52: 6858-6862

43 Hu L, Kim HS, Lee JY, Peumans P, Cui Y. Scalable coating and properties of transparent, flexible, silver nanowire electrodes. ACS Nano, 2010, 4: 2955-2963

44 Sun Y, Yin Y, Mayers BT, Herricks T, Xia Y. Uniform silver nanowires synthesis by reducing $\mathrm{AgNO}_{3}$ with ethylene glycol in the presence of seeds and poly(vinyl pyrrolidone). Chem Mater, 2002, 14: 4736-4745

45 Sun Y, Gates B, Mayers B, Xia Y. Crystalline silver nanowires by soft solution processing. Nano Lett, 2002, 2: 165-168

Acknowledgements This work was supported by the National Natural Science Foundation of China (21471099), and Evonik Industries.

Author Contributions Che S designed the experiment and led the project. Ma L synthesised the chiral Ag nanowires and performed the SEM, XRD and CD measurements and Huang $Z$ obtained the TEM images. Duan Y, Huang $Z$ and Shen $X$ provided comments and feedback on the manuscript. All authors contributed to the general discussions.

Conflict of interest The authors declare that they have no conflict of interest. 


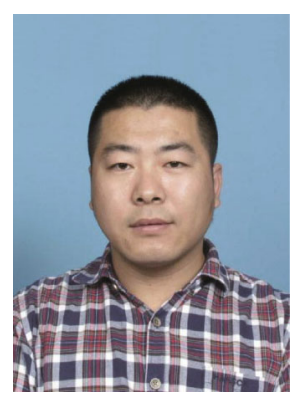

Liguo Ma received his BSc degree in chemical engineering from Changchun University of Technology in 2007. He joined Prof. Che's group as a PhD candidate in 2011. His research interests focus on the synthesis and properties of chiral inorganic materials.

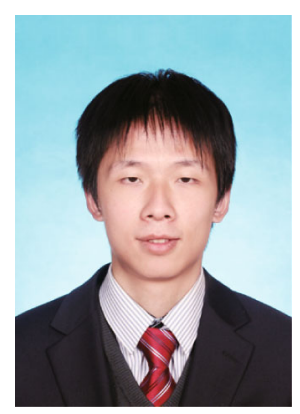

Zhehao Huang obtained his BSc degree in chemistry in 2009, and received his PhD degree under Prof. Che's supervision in 2014 from Shanghai Jiao Tong University. His current research interests include self-assembly of biomolecules, fabrication of bio-inspired materials and nanomaterials, and the corresponding applications.

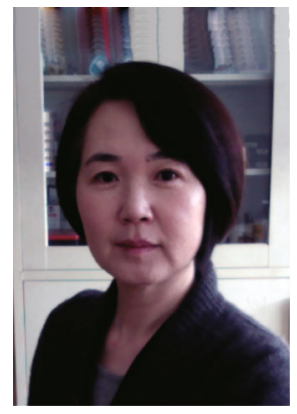

Shunai Che is a professor in the Department of Chemistry, School of Chemistry and Chemical Engineering, Shanghai Jiao Tong University. She was born in 1964 and received her PhD degree from Yokohama National University. She was a guest researcher at Saitama University and worked as a postdoctoral fellow at Yokohama National University. Her research interests encompass the development of chiral inorganic materials and porous materials with novel structures and functions in view of applications in optical devices and heterogeneous catalysis.

中文摘要 本文以手性碳纳米管为模板, 成功地在其内部形成了手性银纳米线. 由于手性排列的银纳米线之间的集合耦合效应,在可见 光区和近红外区产生了较强的手性圆二色信号. 根据我们先前报道的方法, 通过碳化处理自组装合成的手性吡咯碳纳米管得到了单一 手性的碳纳米管. 由于毛细管效应, 银离子能够很容易地进入手性碳纳米管的手性孔道中, 然后再通过氢气高温还原, 在其管内得到了 具有光学活性的手性银纳米线. 手性碳纳米管内的螺旋孔道对手性银纳米线的形成起模板作用. 该合成体系将有助于理解具有手性光 学活性的无机材料的形成及其机理. 这种新颖的手性无机材料也将有机会应用到非线性光学器件、生物传感和手性识别等领域. 